Review Article

\title{
Systematic Review and Meta-Analysis of Oxidative Stress and Antioxidant Markers in Oral Lichen Planus
}

\author{
Jia Wang $\mathbb{D}^{1,2,3}$ Jingjing Yang, ${ }^{1,2,3}$ Chen Wang, ${ }^{4}$ Zhibai Zhao, ${ }^{1,2,3}$ and Yuan Fan $\mathbb{D}^{1,2,3}$ \\ ${ }^{1}$ Department of Oral Medicine, The Affiliated Stomatological Hospital of Nanjing Medical University, Nanjing, \\ 210029 Jiangsu, China \\ ${ }^{2}$ Jiangsu Province Key Laboratory of Oral Diseases, Nanjing, 210029 Jiangsu, China \\ ${ }^{3}$ Jiangsu Province Engineering Research Center of Stomatological Translational Medicine, Nanjing, 210029 Jiangsu, China \\ ${ }^{4}$ College \& Hospital of Stomatology, Anhui Medical University, Key Laboratory of Oral Diseases Research of Anhui Province, \\ Hefei 230032, China
}

Correspondence should be addressed to Yuan Fan; fanyuan@njmu.edu.cn

Received 10 March 2021; Revised 14 August 2021; Accepted 28 August 2021; Published 27 September 2021

Academic Editor: Cristina Nocella

Copyright (c) 2021 Jia Wang et al. This is an open access article distributed under the Creative Commons Attribution License, which permits unrestricted use, distribution, and reproduction in any medium, provided the original work is properly cited.

\begin{abstract}
Background. Oral lichen planus (OLP) is a relatively common chronic inflammatory disease of unknown etiology, which might be caused by oxidative stress and impaired antioxidant defense. Objective. To systematically investigate the markers of oxidative stress and antioxidant systems in the saliva and blood from OLP patients and healthy controls. Methods. The PubMed, Cochrane Library, and Embase were systematically queried to collect data from studies in which oxidative stress/antioxidant markers from OLP and healthy subjects had been evaluated until March 10, 2021. Results. A total of 28 studies fulfilled inclusion criteria, and 25 of them, having 849 OLP patients and 1,052 control subjects and analyzing 12 oxidative stress and antioxidant state marker levels, were subjected to meta-analysis. We found a significant decrease in total antioxidant capacity (TAC) and uric acid (UA) and a significant increase in malondialdehyde (MDA) and nitric oxide (NO) levels in the saliva and serum/plasma of OLP patients. Moreover, a significant elevation of 8-hydroxy-deoxyguanosine (8-OHdG) and advanced oxidation protein product (AOOP) level and a decrease in vitamin $\mathrm{C}$ were also observed in the saliva of the OLP group. In contrast, salivary vitamin A, zinc, glutathione peroxidase (GPx), vitamin E, and nitrite were not significantly different between the two groups. In single studies, markers of oxidative stresses such as superoxide dismutase (SOD) and 8-isoprostanelevels were elevated in OLP, and antioxidant parameters such as glutathione (GSH) and total protein (TP) levels were dysregulated. Conclusion. This meta-analysis helps to clarify the profile of oxidative stress and antioxidant state markers in OLP patients although existing evidence is rather heterogeneous and many studies are affected by several limitations. Larger and more standardized studies are warranted to ascertain whether these markers are potential causes or effects of OLP and whether antioxidant therapy improving oxidative stress will be useful.
\end{abstract}

\section{Introduction}

Oral lichen planus (OLP), a chronic inflammatory disease characterized by relapses and remissions, could be found in about $2 \%$ of people all around the world, with an overall age-standardized global prevalence of $1.27 \%$ ( $0.96 \%$ in men and $1.57 \%$ in women) in the general population [1], more commonly found in women aged between 50 and 60 years [2]. OLP is a precancerous lesion, and a malignant transformation varies between $0 \%$ and $12.5 \%$ [3]. Although its etiol- ogy is unknown, it is believed to be mediated by an immune disorder [4]. Therefore, more extensive knowledge and more accurate diagnostic tools of OLP are particularly important.

Recent years have seen increasing research interest in oxidative stress (OS) in the pathogenesis of several diseases, such as inflammatory, chronic degenerative (Alzheimer's disease), cardiovascular, or autoimmune [5-8]. OS modifies the normal intracellular balance, producing excessive oxidant substances, i.e., reactive oxygen species (ROS) and reactive nitrogen species (RNS) and resulting in a relative 
deficiency of enzymatic and nonenzymatic antioxidants [9, 10]. The members of antioxidant systems are currently used as "indirect" biomarkers of oxidative stress generation [11]. Enzymatic antioxidant systems comprise superoxide dismutase (SOD), catalase (CAT), and glutathione peroxidase (GSHPx) [12, 13]. Nonenzymatic antioxidant systems include minerals and vitamins [14].

OS may play a central role in the etiology of OLP [15]. The subepithelial infiltration of T lymphocytes in OLP contributes to the local production of cytokines, which can stimulate production of ROS [16]. The presence of apoptosis is a hallmark feature in OLP. ROS are essential mediators of apoptosis and may result in the dysfunction of keratinocytes and their impaired apoptosis [17].

A recent study provided a complete review of the association of micronutrients with OLP, suggesting a decrease in antioxidant levels and an increase in oxidant levels in OLP patients [18], both being significant. In line with these findings, increased nitric oxide (NO) and malondialdehyde (MDA) have also been found in patients with OLP and suggested as biomarkers for monitoring disease activity and therapeutic responses in OLP $[19,20]$. Overall, these studies show that OLP patients are more susceptible to oxidantantioxidant imbalance. To date, however, there is no consensus on the most appropriate biomarkers of oxidative stress and antioxidant systems in OLP. No quantitative systematic meta-analysis has been conducted on this subject either. Therefore, we aimed to perform a meta-analysis to quantify the association between OLP and these markers.

\section{Methods}

2.1. Protocol and Registration, Search Strategy, and Eligibility Criteria. The protocol was registered at PROSPERO (CRD42020199851) in accordance with the Cochrane Handbook [21] and PRISMA guidelines [22]. The review focused on the alterations of oxidative stress and antioxidant barrier markers in the saliva or serum with and without OLP.

We conducted an electronic literature search using PubMed, Cochrane Library, and Embase until 03/10/2021 for studies comparing oxidative stress and antioxidant markers between OLP patients and healthy controls. Controlled vocabulary terms (MeSH) and the following keywords were used in the search strategy: (lichen planus, oral [MeSH Terms]OR oral lichen planus OR OLP) AND (( $(\mathrm{ROS}$ OR reactive oxygen species $\mathrm{OR}$ reactive nitrogen species OR free radicals OR oxidized LDL OR protein carbonylation OR lipid peroxidation OR MDA OR malondialdehyde OR thiobarbituric acid reactive substances OR asymmetric dimethylarginine OR 8-OHdG OR 8-hydroxyguanosine OR homocysteine OR SOD OR superoxide dismutase OR glutathione OR GPX OR glutathione peroxidase OR glutathione reductase OR catalase OR vitamin A OR vitamin $\mathrm{C}$ OR vitamin $\mathrm{E}$ OR a-carotene OR b-carotene OR ascorbic acid OR paraoxonase OR albumin OR uric acid OR high-density lipoprotein cholesterol OR zinc OR NO OR nitric oxide OR nitrite OR peroxide OR 8-F2isoprostane OR protein carbonyl) OR (antioxidants [MeSH Terms] OR anti-oxidants OR anti oxidants OR anti- oxidant effect OR anti oxidant effect OR anti-oxidant effects OR anti oxidant effects OR total antioxidant* OR antioxidative stress $\mathrm{OR}$ antioxidant* $\mathrm{OR}$ antinitrative stress)) OR (oxidative stress [MeSH Terms] OR oxidative stresses OR stresses, oxidative OR stress, oxidative OR oxidation OR oxidative damage OR nitrative damage OR oxidant* OR total oxidant*)). The search was not limited to specific years. An English language restriction was imposed. Gray literature was searched in OpenGrey.

The inclusion criteria were as follows: (a) population-patients were clinically and histopathologically diagnosed with oral lichen planus; (b) exposure-at least one oxidative stress or antioxidant marker was measured in the saliva or serum/plasma sample; (c) comparators-the article included both the healthy control group and OLP group; (d) outcomes-sufficient information regarding the levels of oxidative stress and antioxidant system markers was presented for further analysis; and (e) study designs - the research was designed as either a case-control study or a cross-sectional survey.

2.2. Data Collection and Quality Assessment. After independent searches, titles and abstracts were reviewed by 2 reviewers (J.W. and JJ. Y) who then read the full texts to determine the final eligibility. Discrepancies were resolved through consensus or involvement of a third author (Y.F.). One author (J.W.) extracted all the data, which were independently verified by another author (JJ. Y). For studies that did not provide specific data, we contacted the authors to request their original data. If no response was received, the reviewers excluded their studies. Data extracted from each eligible study included the following: (a) article information-first author, year, country, journal, title, study design, and sample size; (b) participant characteristics-age, sex, and types of OLP; and (c) outcomes-mean \pm SD or median (IQR), median (min-max) of OS and antioxidant biomarker levels (for both OLP patients and controls), and sample source.

Quality scores of case-control (CC) studies were assessed with the Newcastle-Ottawa scale (NOS), which was based on 3 topics: selection, comparability, and exposures (11 items in total, one star for each) [23]. Studies with 7 to 9 stars were recognized having high quality, those with 5 to 6 stars as medium quality, and those with $<5$ stars as low quality. The quality of cross-sectional (CS) survey was evaluated by the Agency for Healthcare Research and Quality (AHRQ) (one point for each) [24]. The qualities of studies with 0-3 points were recognized as low, those with 4-7 points as moderate, and those with $8-11$ points as high. The Review Manager 5.4 special software for the Cochrane collaborative network was used to output the result of NOS and AHRQ.

2.3. Statistical Analysis. Once more than two studies reported a similar biomarker, we would conduct metaanalysis. The sample size and mean \pm standard deviation (SD) were used to generate the effective size. The standardized mean difference (SMD) was calculated because of some clinical and methodological heterogeneity between studies. The $95 \%$ confidence interval (95\% CI) was used to estimate 


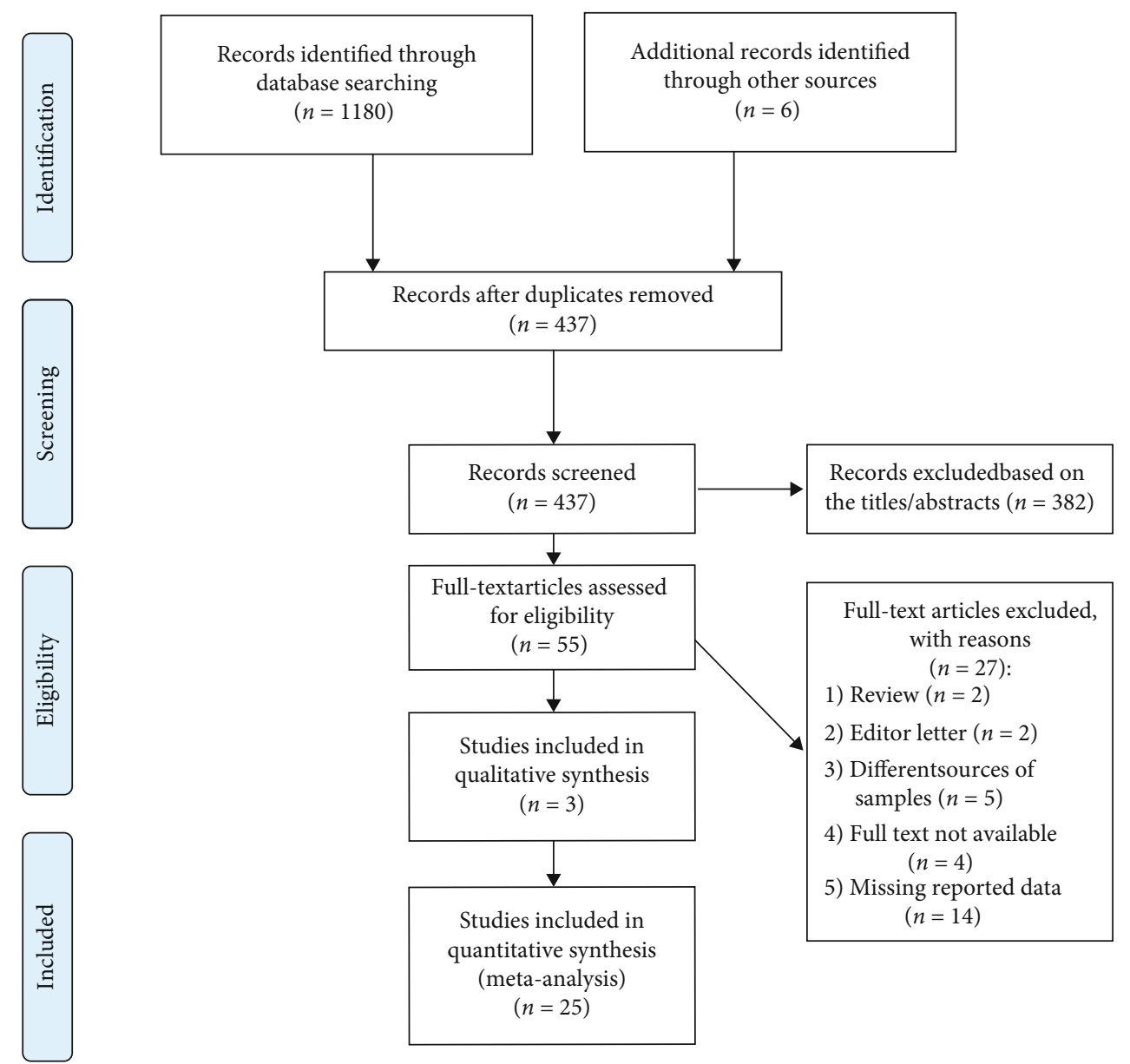

FIGURE 1: PRISMA flow diagram of the study selection process.

the statistical significance of the pooled effective size. $P<$ 0.05 was considered statically significant. To compare parallel studies, a method described by Hozo et al. [25] was used to calculate an estimate of the correspondent mean and SD if the data were reported as median (IQR) or median (min$\max )$. We assessed statistical heterogeneity using the $I^{2}$ statistic. When $I^{2}>50 \%$, we used a random effects model to calculate effect size; otherwise, the fixed effects model was used [26]. Values of 25\%,50\%, and 75\% indicate low, moderate, and high heterogeneity, respectively.

When the number of included articles was more than 6 and $I^{2}>50 \%$, we further conducted subgroup analysis to explore possible explanation for heterogeneity and sensitivity analysis to confirm the robustness of the main findings. A subgroup analysis was done as categorized by race (Asian vs. European), and the sensitivity analysis was performed by removing the studies one by one. If the number of included studies was smaller than 10, visual analysis of funnel plots was not very reliable [27]. All data were analyzed using Review Manager 5.4 (the Nordic Cochrane Center, the Cochrane Collaboration, Copenhagen, Denmark).

\section{Results}

3.1. Literature Searches. The flow diagram (Figure 1) presented the review selection process and the study selection process. We retrieved 1,186 records published prior to 03/10/2021 from the databases. After excluding duplicates, 437 records were filtered based on title and abstract. The full text of 55 of these records was retrieved for further assessment. After the full texts were read, 27 articles were excluded. In total, 28 articles fulfilled the inclusion criteria and 25 studies were eligible for our meta-analysis.

3.2. Study Characteristics. A total of 25 eligible studies were illustrated in detail in Table 1, which involved 849 OLP patients and 1,052 control subjects in the meta-analysis. Totally, 12 oxidative stress and antioxidant barrier markers were adopted in this meta-analysis. We present a summary of the findings of our meta-analyses in Table 2, which includes the number of studies/participants, the corresponding summary effect size, and $I^{2}$ value for each meta-analysis. Figures 2-5 show forest plots of the oxidative stress, nitrosative stress, antioxidant barrier (enzymatic and nonenzymatic), and total redox status markers with the significant difference between OLP and healthy controls, respectively. All the studies had a cross-sectional and case-control design; patients were clinically/histopathologically evaluated for OLP. According to the assessment by the NOS and AHRQ checklist (Figure 6), we assigned a low quality to 3 studies and a moderate quality to 2 studies among the 5 crosssectional studies. In 20 case-control studies, 3 were of high 


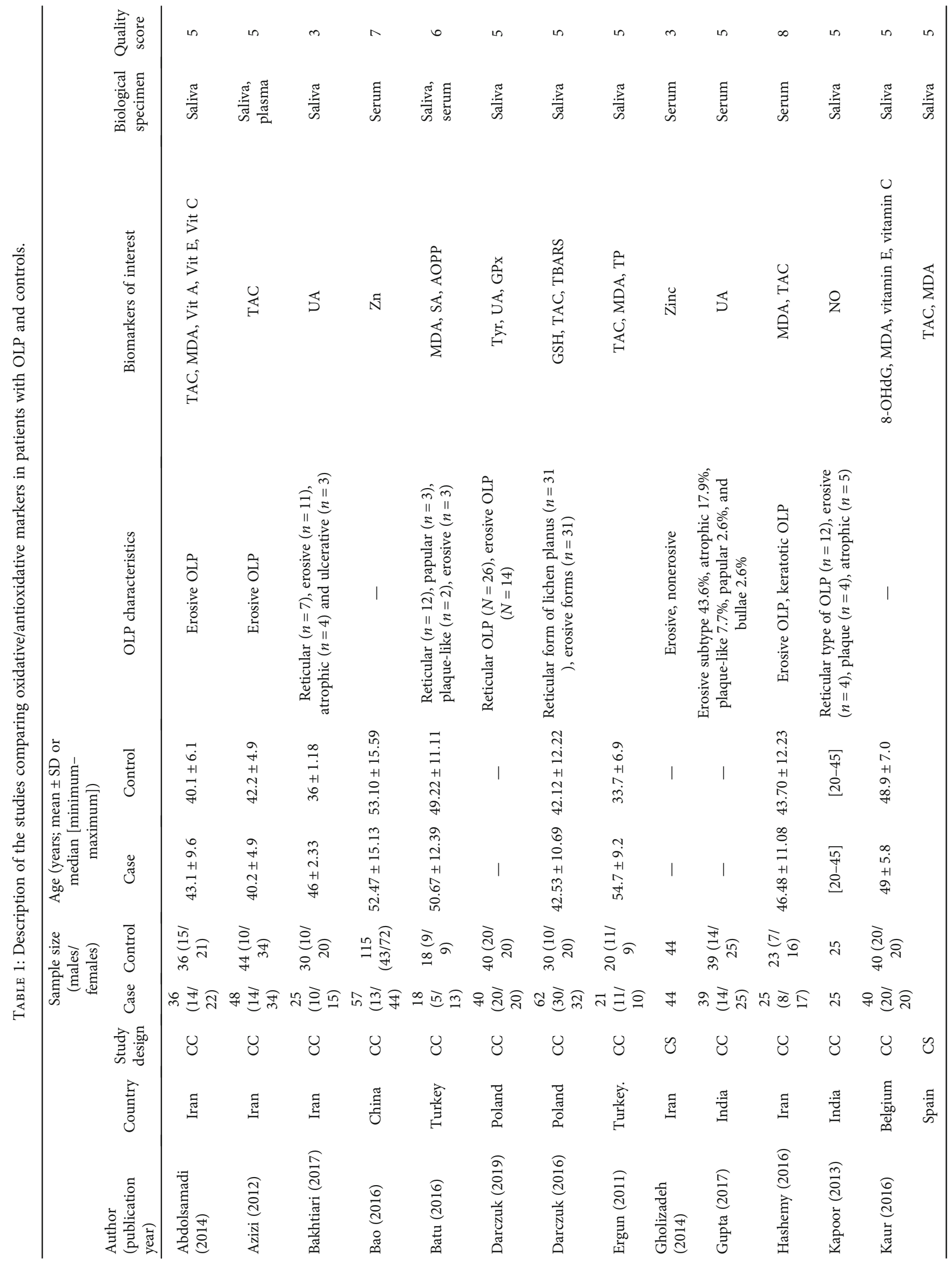




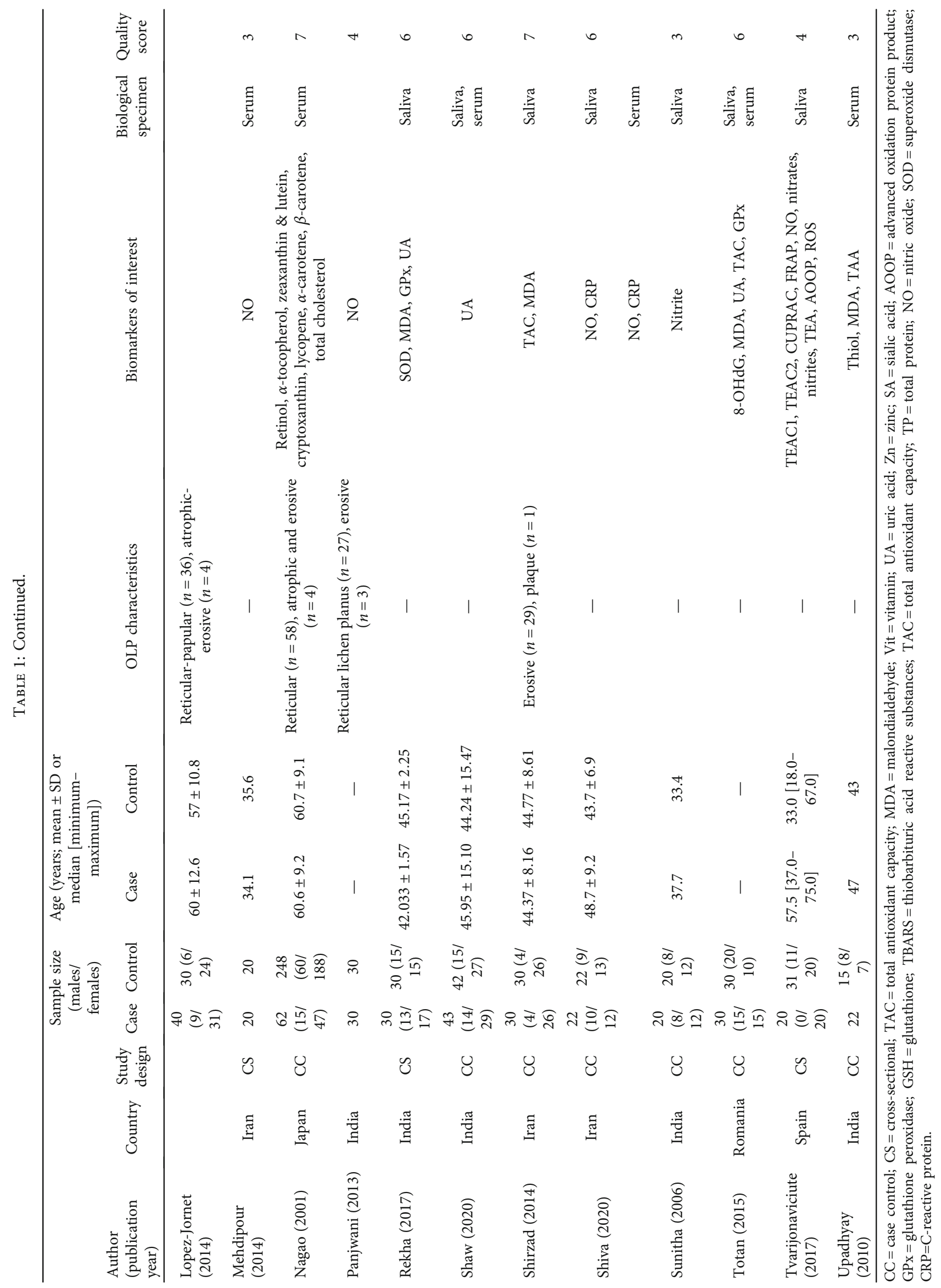


TABLE 2: Meta-analyses of comparisons of oxidative/antioxidative markers between OLP patients and controls.

\begin{tabular}{|c|c|c|c|c|c|c|c|c|c|c|}
\hline \multirow[b]{2}{*}{ Marker (source) } & \multirow[b]{2}{*}{ \# of studies } & \multirow[b]{2}{*}{ OLP, $N$} & \multirow[b]{2}{*}{ Control, $N$} & \multicolumn{3}{|c|}{ Effect size (OLP vs. controls) } & \multicolumn{4}{|c|}{ Heterogeneity } \\
\hline & & & & SMD $(95 \% \mathrm{CI})$ & $Z$ value & $P$ value* & $\mathrm{Chi}^{2}$ & df & $P$ value & $I^{2}(\%)$ \\
\hline NO (saliva) & 4 & 97 & 108 & $3.25(1.11,5.39)$ & 2.98 & $P=0.003$ & 86.4 & 3 & $P<0.00001$ & 97 \\
\hline NO (serum/plasma) & 2 & 42 & 42 & $1.07(0.60,1.53)$ & 4.47 & $P<0.00001$ & 1.03 & 1 & $P=0.31$ & 3 \\
\hline MDA (saliva) & 9 & 307 & 264 & $2.38(1.47,3.29)$ & 5.13 & $P<0.00001$ & 138.81 & 8 & $P<0.00001$ & 94 \\
\hline MDA (serum/plasma) & 4 & 91 & 83 & $0.92(0.38,1.46)$ & 3.34 & $P=0.0008$ & 8.37 & 3 & $P=0.04$ & 64 \\
\hline 8-OHdG (saliva) & 2 & 70 & 70 & $3.78(0.14,7.42)$ & 2.04 & $P=0.04$ & 38.24 & 1 & $P<0.00001$ & 97 \\
\hline AOOP (saliva) & 2 & 39 & 49 & $1.00(0.54,1.45)$ & 4.31 & $P<0.00001$ & 0.50 & 1 & $P=0.48$ & 0 \\
\hline TAC (saliva) & 7 & 267 & 220 & $-2.03(-3.03,-1.03)$ & 3.98 & $P<0.0001$ & 115.29 & 6 & $P<0.00001$ & 95 \\
\hline TAC (serum/plasma) & 4 & 121 & 109 & $-2.87(-4.56,-1.19)$ & 3.34 & $P=0.0008$ & 57.84 & 3 & $P<0.00001$ & 95 \\
\hline Vit C (saliva) & 2 & 76 & 76 & $-2.03(-3.16,-0.89)$ & 3.5 & $P=0.0005$ & 7.87 & 1 & $P=0.005$ & 87 \\
\hline UA (saliva) & 5 & 168 & 173 & $-2.65(-4.20,-1.09)$ & 3.33 & $P=0.00009$ & 127.97 & 4 & $P<0.00001$ & 97 \\
\hline UA (serum/plasma) & 3 & 112 & 111 & $-1.19(-1.83,-0.54)$ & 3.61 & $P=0.0003$ & 9.77 & 2 & $P=0.008$ & 80 \\
\hline Vit A (saliva) & 2 & 98 & 284 & $-0.86(-3.24,1.52)$ & 0.71 & $P=0.48$ & 54.52 & 1 & $P<0.00001$ & 98 \\
\hline Zn (serum/plasma) & 2 & 101 & 159 & $0.21(-1.73,2.16)$ & 0.22 & $P=0.83$ & 47.99 & 1 & $P<0.00001$ & 98 \\
\hline GPx (saliva) & 3 & 100 & 100 & $-1.34(-2.81,0.13)$ & 1.78 & $P=0.07$ & 42.02 & 2 & $P<0.00001$ & 95 \\
\hline Vit E (saliva) & 3 & 138 & 324 & $-1.53(-3.41,0.34)$ & 1.60 & $P=0.11$ & 92.75 & 2 & $P<0.00001$ & 98 \\
\hline Nitrite (saliva) & 2 & 40 & 51 & $-0.23(-2.81,2.35)$ & 0.17 & $P=0.86$ & 30.43 & 1 & $P<0.00001$ & 97 \\
\hline
\end{tabular}

$\mathrm{TAC}=$ total antioxidant capacity; Vit $\mathrm{C}=$ vitamin $\mathrm{C} ; \mathrm{UA}=$ uric acid; $\mathrm{NO}=$ nitric oxide; $\mathrm{MDA}=$ malondialdehyde; $\mathrm{AOOP}=$ advanced oxidation protein product; Vit $\mathrm{A}=$ vitamin $\mathrm{A} ; \mathrm{Zn}=$ zinc; $\mathrm{GPx}=$ glutathione peroxidase; Vit $\mathrm{E}=$ vitamin $\mathrm{E}$.

quality, 4 were of low quality, and the remaining 10 were of medium quality.

\subsection{Oxidative Stress Markers in OLP}

3.3.1. Lipid Peroxidation Malondialdehyde (MDA). Three studies $[15,28,29]$ demonstrated significantly higher MDA in the serum/plasma from OLP patients while one study [30] mentioned no difference. Overall, MDA in the serum/plasma showed a significant increase in OLP (effect size 0.92, $95 \%$ CI $0.38,1.46)$, with statistically significant heterogeneity $\left(P=0.04, I^{2}=64 \%\right)$. MDA was measured in the saliva from OLP in nine studies and found to be markedly elevated in all nine (effect size $2.38,95 \%$ CI 1.47, 3.29), with statistically significant heterogeneity $\left(P<0.00001, I^{2}=94 \%\right) \quad[16$, 28-35].

3.3.2. 8-Hydroxy-deoxy (8-OHdG). For evidence of oxidative DNA/RNA damage, $8-\mathrm{OHdG}$ in the saliva from OLP patients was evaluated in two studies (70 patients) and found to be increased in both under a random effects model (effect size $3.78,95 \%$ CI $0.14,7.42$ ), with statistically significant heterogeneity $\left(P<0.00001, I^{2}=97 \%\right)[29,33]$. Totan et al. also evaluated 8 -OHdG in the serum and found a significant increase in OLP patients [29].

3.3.3. Advanced Oxidation and Products (AOPP). Only two studies (38 patients) evaluated AOPP levels in the saliva from patients with OLP under a fixed effects model; both showed a significant increase (effect size $1.00,95 \%$ CI 0.54 , $1.45)$, with no significant heterogeneity $\left(P=0.48, I^{2}=0 \%\right)$
$[28,36]$. In a single study, increased AOPP was reported in the serum from OLP patients [28].

\subsection{Nitrosative Stress Markers in OLP}

3.4.1. Nitric Oxide (NO). As for the NO level in saliva, all the four original studies (97 patients) demonstrated significantly higher NO level in OLP than in controls (effect size 3.25, 95\% CI 1.11, 5.39) [36-39]. The same results were also presented in serum/plasma among two studies (effect size 1.07, 95\% CI $0.60,1.53)[39,40]$. The heterogeneity of NO in saliva was significant $\left(P<0.00001, I^{2}=94 \%\right)$, while NO in serum/plasma had no significant heterogeneity $\left(P=0.31, I^{2}=3 \%\right)$.

3.4.2. Nitrite. Nitrite in saliva was evaluated in 2 studies, and contrast results were achieved. Lower nitrite in saliva was found by Sunitha et al. [41], whereas Tvarijonaviciute et al. showed a much higher level in OLP patients than in healthy controls [36]. Meta-analyzing data of nitrite in saliva revealed no significant difference in cases and controls.

\subsection{Antioxidant Barrier in OLP}

\subsubsection{Enzymatic Antioxidant}

(1) Glutathione Peroxidase (GPx). In saliva, three studies all reported significantly lower GPx in OLP cases, while there was no significant association of GPx with OLP across the meta-analyses [29, 35, 42]. In addition, GPx in serum [29] (30 patients), thiol in serum [15] (22 patients), and GSH in saliva [32] (62 patients) were determined in the single study, and decreased levels were found. 


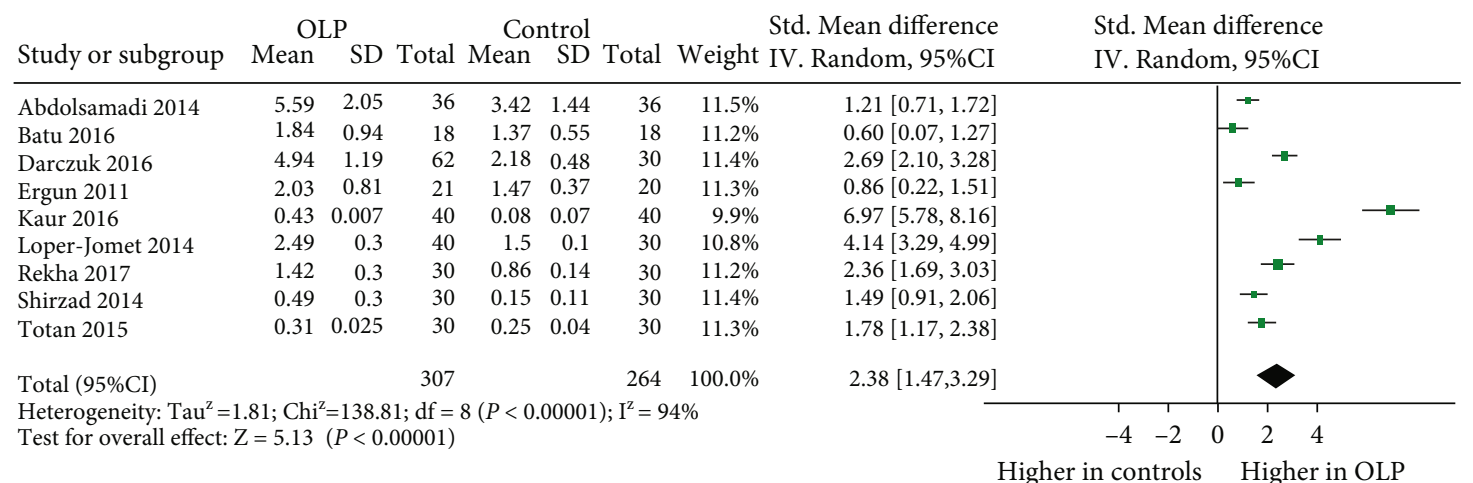

(a) $\operatorname{MDA}($ saliva)

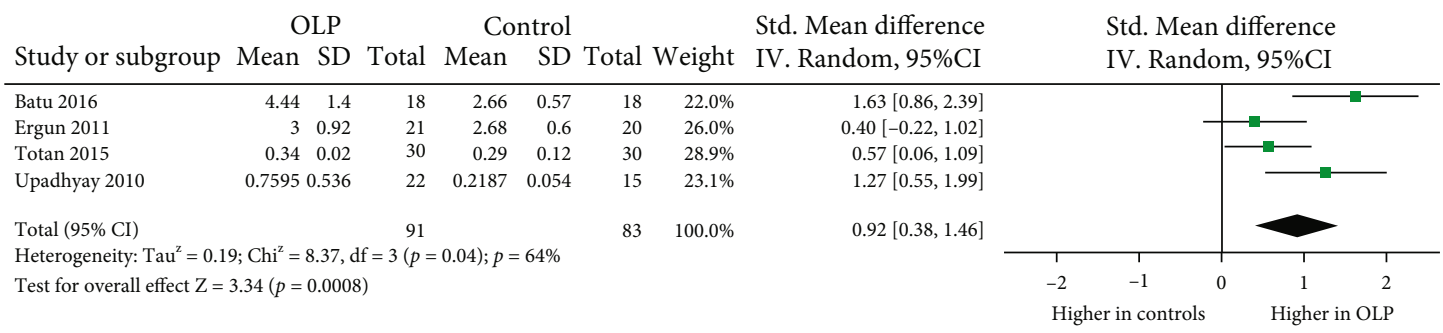

(b) MDA(serum/plasma)

\begin{tabular}{|c|c|c|c|c|c|c|c|c|c|c|c|c|c|}
\hline Study or subgroup & Mean & $\begin{array}{l}\text { LP } \\
\text { SD }\end{array}$ & Total & $\begin{array}{l}\text { Con } \\
\text { Mean }\end{array}$ & $\begin{array}{l}\text { trol } \\
\text { SD }\end{array}$ & \multicolumn{2}{|c|}{ Total Weight } & $\begin{array}{l}\text { Std.Mean difference } \\
\text { IV.Random, 95\% CI }\end{array}$ & \multicolumn{5}{|c|}{$\begin{array}{l}\text { Std.Mean difference } \\
\text { IV.Random, 95\% CI }\end{array}$} \\
\hline Kaur 2016 & 0.47 & 0.07 & 40 & 0.07 & 0.07 & 40 & $49.4 \%$ & $5.66[4.66,6.66]$ & & & & - & \\
\hline Totan 2015 & 10.5 & 2.74 & 30 & 6.47 & 0.93 & 30 & $50.6 \%$ & $1.94[1.32,2.57]$ & & & & & \\
\hline $\begin{array}{l}\text { Total }(95 \% \mathrm{CI}) \\
\text { Heterogenity.Tau }{ }^{2}=6.72 \text {; }\end{array}$ & $\mathrm{Chi}^{2}=38$ & $3.24, \mathrm{df}$ & $\begin{aligned} & 70 \\
= & 1(P<\end{aligned}$ & $0.00001)$ & $I^{2}=97$ & 70 & $100.0 \%$ & $3.78[0.14,7.42]$ & & & & & \\
\hline Test for overall effect $Z=$ & $2.04(P=$ & $0.04)$ & & & & & & & -10 & -5 & 0 & 5 & 10 \\
\hline
\end{tabular}

(c) 8-OHdG(saliva)

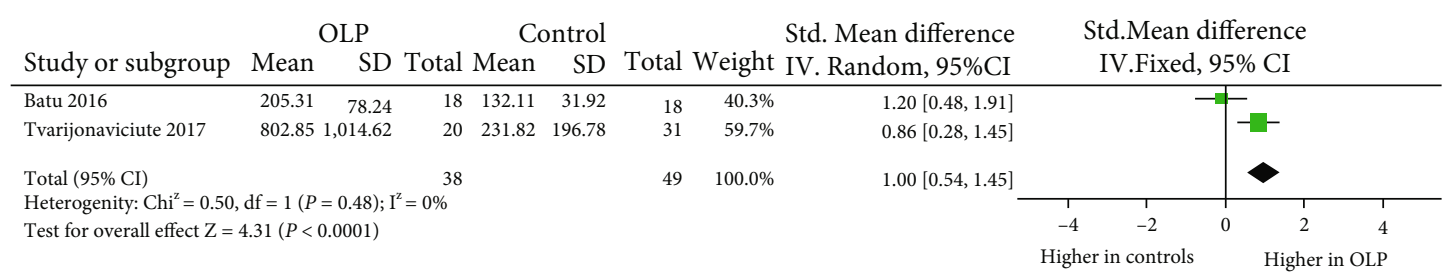

(d) $\mathrm{AOOP}$ (saliva)

FIGURE 2: Evidence of increased oxidative stress markers in oral lichen planus (OLP). Forest plots show higher malondialdehyde (MDA) in saliva and serum/plasma and 8-hydroxy-deoxyguanosine (8-OHdG) and advanced oxidation protein product (AOOP) level in saliva from subjects with OLP compared to healthy controls $(P<0.05$ for each).

\subsubsection{Nonenzymatic Antioxidant}

(1) Uric Acid (UA). UA in plasma/serum was investigated in three studies and found to be markedly decreased in OLP compared to controls (effect size -1.19, 95\% CI -1.83, $-0.54)$, with statistically significant heterogeneity $(P=0.008$, $\left.I^{2}=80 \%\right)[29,43,44]$. Four studies reported lower salivary UA in OLP cases [29, 42, 44, 45], while one single study did not report any statistically significant difference between OLP and controls [35]. The meta-analysis for UA assessed in saliva was -2.65 (95\% CI $-4.20,-1.09)$, with statistically significant heterogeneity $\left(P<0.00001, I^{2}=97 \%\right)$.
(2) Zinc ( $Z n$ ). Contrasting data were reported from two studies on $\mathrm{Zn}$ levels in serum/plasma. Bao et al. showed a much lower $\mathrm{Zn}$ in the saliva of OLP patients [46], while the results were opposite in the other study by Gholizadeh et al. [47]. The meta-analysis for $\mathrm{Zn}$ in serum/plasma showed no significant difference between OLP and controls. Only one study evaluated $\mathrm{Zn}$ in saliva and showed no significant difference between OLP and healthy controls [48].

(3) Vitamins. Concerning vitamin A, one study with 36 OLP patients did not observe a difference while a large study with 62 OLP patients detected significantly higher vitamin A in 


\begin{tabular}{|c|c|c|c|c|c|c|c|c|c|c|c|c|}
\hline \multirow[b]{2}{*}{ Study or subgroup } & \multicolumn{2}{|c|}{ OLP } & \multicolumn{3}{|c|}{ Control } & \multirow[b]{2}{*}{ Total } & \multicolumn{2}{|c|}{ Std. Mean difference } & \multicolumn{3}{|c|}{ Std. Mean difference } & \multirow[b]{3}{*}{ 一— } \\
\hline & Mean & $\mathrm{SD}$ & Total & Mean & $\mathrm{SD}$ & & Weight & IV. Random, 95\%CI & \multicolumn{3}{|c|}{ IV. Random, 95\%CI } & \\
\hline Kapoor 2013 & 90.88 & 12.056 & 25 & 13.84 & 3.566 & 25 & $22.3 \%$ & $8.53[6.70,10.36]$ & & & & \\
\hline Panjwani 2013 & 60.9 & 21.86 & 30 & 7.2 & 2.96 & 30 & $25.6 \%$ & $3.40[2.59,4.21]$ & & & - & \\
\hline Shiva 2020 & 84 & 37.66 & 22 & 66.75 & 23.66 & 22 & $26.1 \%$ & $0.54[-0.06,1.14]$ & & & + & \\
\hline Tvarijonaviciute 2017 & 196.89 & 174.33 & 20 & 40.6 & 60.36 & 31 & $26.0 \%$ & $1.30[0.68,1.92]$ & & & - & \\
\hline Total $(95 \% \mathrm{CI})$ & & & 97 & & & 108 & $100.0 \%$ & $3.25[1.11,5.39]$ & & & & \\
\hline \multicolumn{9}{|c|}{$\begin{array}{l}\text { Heterogeneity: } \mathrm{Tau}^{2}=4.48 ; \mathrm{Chi}^{2}=86.40, \mathrm{df}=3(P<0.00001): \mathrm{I}^{\mathrm{z}}=97 \% \\
\text { Test for overall effect: } \mathrm{Z}=2.98(P=0.003)\end{array}$} & -10 & -5 & 0 & 10 \\
\hline \multicolumn{9}{|c|}{ Test for overall effect: $\mathrm{Z}=2.98(P=0.003)$} & Higher & 0 & & er in OLP \\
\hline
\end{tabular}

(a) $\mathrm{NO}$ (saliva)

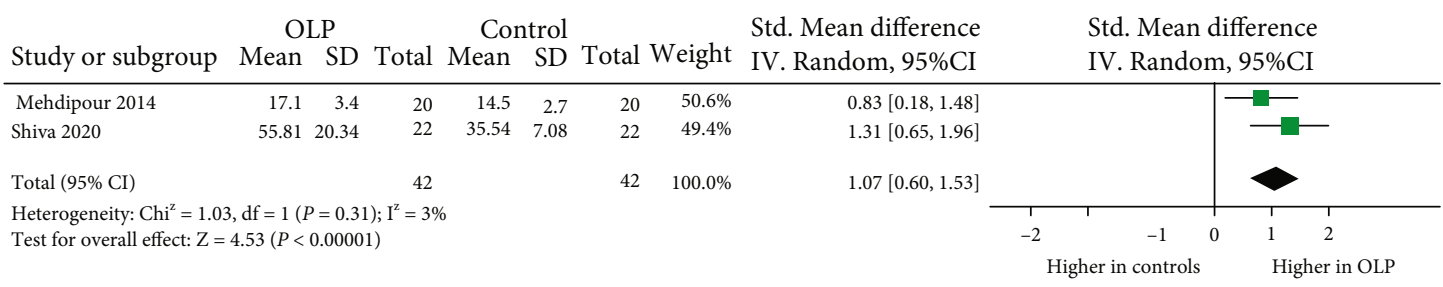

(b) $\mathrm{NO}$ (serum/plasma)

FIGURE 3: Evidence of increased nitrosative stress markers in oral lichen planus (OLP). Forest plots show higher nitric oxide (NO) levels in saliva and serum/plasma from OLP patients compared to healthy controls $(P<0.05$ for each).

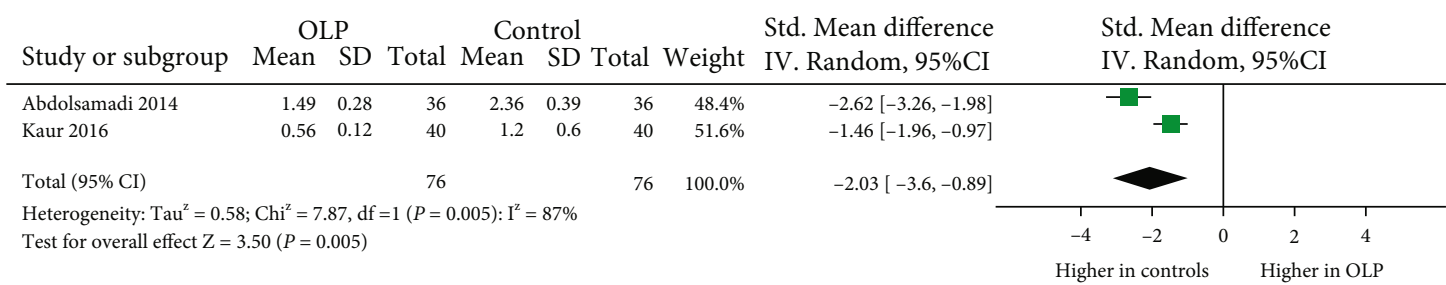

(a) Vit C(saliva)

\begin{tabular}{|c|c|c|c|c|c|c|c|c|c|c|c|c|}
\hline Study or subgroup & \multicolumn{3}{|c|}{ OLP } & $\begin{array}{l}\text { Cor } \\
\text { Mean }\end{array}$ & $\begin{array}{r}\text { trol } \\
\text { SD }\end{array}$ & Total & Weight & $\begin{array}{l}\text { Std. Mean difference } \\
\text { IV. Random, 95\%CI }\end{array}$ & \multicolumn{4}{|c|}{$\begin{array}{l}\text { Std. Mean difference } \\
\text { IV. Random, 95\%CI }\end{array}$} \\
\hline Bakhtiari 2017 & 2.1 & 0.19 & 25 & 4.8 & 0.29 & 30 & $15.4 \%$ & $-10.66[-12.79,-8.52]$ & $=$ & & & \\
\hline Darczuk 2019 & 3.41 & 0.11 & 40 & 3.67 & 0.11 & 40 & $21.1 \%$ & $-2.34[-2.92,-1.77]$ & & - & & \\
\hline Rekha 2017 & 4.05 & 1.45 & 30 & 3.89 & 1.21 & 30 & $21.2 \%$ & $0.12[-0.39,0.62]$ & & & & \\
\hline Shaw 2020 & 5.25 & 1.61 & 43 & 6.18 & 1.28 & 42 & $21.3 \%$ & $-0.63[-1.07,-0.20]$ & & $-\mathbf{m}^{-1}$ & & \\
\hline Totan 2015 & 1.7 & 0.6 & 30 & 3.12 & 0.85 & 30 & $21.0 \%$ & $-1.91[-2.52,-1.29]$ & & - & & \\
\hline Total $(95 \% \mathrm{CI})$ & & & 168 & & & 172 & $100.0 \%$ & $-2.65[-4.20,-1.09]$ & & & & \\
\hline \multicolumn{9}{|c|}{ Heterogeneity: $\mathrm{Tau}^{\mathrm{z}}=2.91 ; \mathrm{Chi}^{2}=127.97, \mathrm{df}=4(P<0.00001) ; \mathrm{I}^{2}=97 \%$} & 1 & 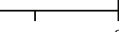 & 1 & \\
\hline \multicolumn{8}{|c|}{ Test for overall effect: $Z=3.33(P=0.0009)$} & & -10 & -5 & 5 & 10 \\
\hline
\end{tabular}

(b) UA(saliva)

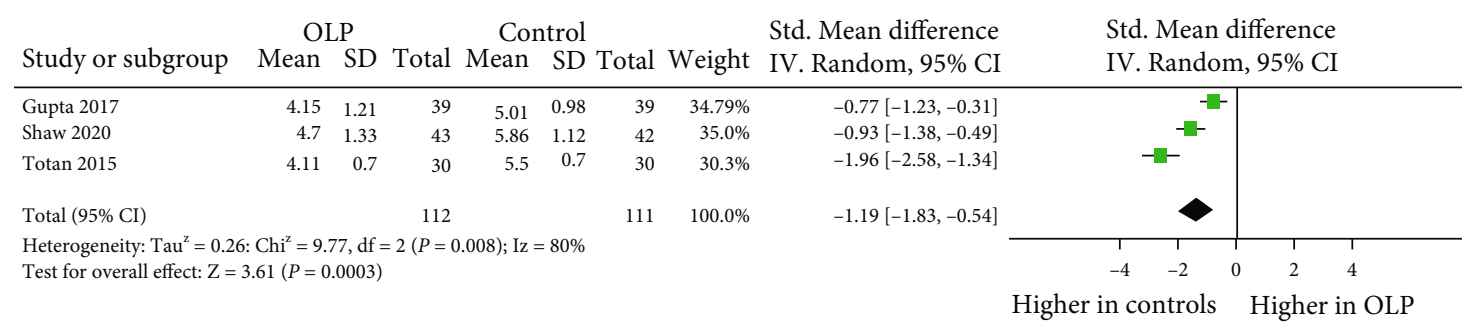

(c) UA(serum/plasma)

Figure 4: Evidence of decreased antioxidant defenses in oral lichen planus (OLP). Forest plots show lower levels of uric acid (UA) in saliva and plasma/serum and vitamin $C$ in saliva from OLP patients to healthy controls $(P<0.05$ for each).

saliva $[26,49]$. As for vitamin C, both of two studies (76 patients) were conducted showing lower vitamin $\mathrm{C}$ in the saliva from OLP $[31,33]$. In the case of vitamin E, two studies $[31,33]$ (76 patients) observed decreased vitamin E levels in saliva while a single study [49] (62 patients) detected no significant difference between patients with OLP and controls. Overall, the levels of vitamin $\mathrm{C}$ in saliva had a significant decrease in OLP (effect size -2.03, 95\% CI -3.16, 


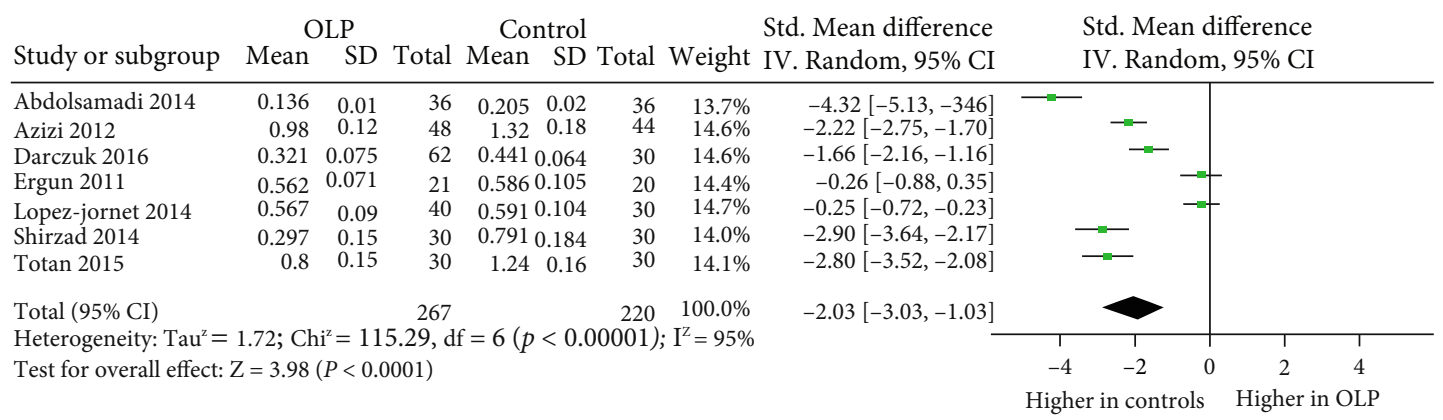

(a) TAC(saliva)

\begin{tabular}{|c|c|c|c|c|c|c|c|c|c|c|c|c|}
\hline Study or subgroup & Mean & SD & Total & $\begin{array}{l}\text { Cor } \\
\text { Mean }\end{array}$ & ntrol & Total & Weight & $\begin{array}{l}\text { Std. Mean difference } \\
\text { IV. Random, 95\% CI }\end{array}$ & \multicolumn{4}{|c|}{$\begin{array}{l}\text { Std. Mean difference } \\
\text { IV. Random, } 95 \% \text { CI }\end{array}$} \\
\hline Azizi 2012 & 1.24 & 0.17 & 48 & 1.84 & 0.34 & 44 & $26.1 \%$ & $-2.24[-2.77,-1.72]$ & $-1-$ & & & \\
\hline Ergun 2011 & 0.716 & 0.078 & 21 & 0.796 & 0.153 & 20 & $25.8 \%$ & $-0.65[-1.28,-0.02]$ & & - & & \\
\hline Totan 2015 & 1.03 & 0.13 & 30 & 1.76 & 0.16 & 30 & $24.3 \%$ & $-4.94[-5.99,-3.90]$ & —- & & & \\
\hline Upadhyay 2010 & 1.054 & 0.3013 & 22 & 2.0370 & 0.1382 & 15 & $23.9 \%$ & $-3.86[-5.00,-2.72]$ & —는 & & & \\
\hline Total $(95 \% \mathrm{CI})$ & & & 121 & & & 109 & $100.0 \%$ & $-2.87[-4.56,-1.19]$ & & & & \\
\hline $\begin{array}{l}\text { Heterogeneity: } \mathrm{Tau}^{2}=2.7 \\
\text { Test for overall effect: } Z=\end{array}$ & $\begin{array}{l}7 ; \mathrm{Chi}^{\mathrm{z}}=5 \\
3.34(P=\end{array}$ & $\begin{array}{r}57.84, \mathrm{df} \\
0.0008)\end{array}$ & $=3(P<C$ & 0.00001); & $I^{z}=95 \%$ & & & & -4 & -2 & 2 & 4 \\
\hline
\end{tabular}

(b) TAC(serum/plasma)

FIGURE 5: Evidence of decreased total redox status in oral lichen planus (OLP). Forest plots show lower levels of total antioxidative capacity (TAC) in saliva and plasma/serum from OLP patients than from healthy individuals $(P<0.05$ for each).

$-0.89)$, with statistically significant heterogeneity $(P=0.005$, $I^{2}=87 \%$ ), while there were no significant differences of salivary vitamin $\mathrm{E}$ and vitamin A between OLP and controls.

3.5.3. Others. Antioxidant nutrients and trace elements counteract free radical damage and thereby protect cell membranes against lipid peroxidation. Magnesium $(\mathrm{Mg})$, copper $(\mathrm{Cu})$, vitamin B12, folic acid, Tyr, TP, thiol, cryptoxanthin, lycopene, and nitrite were only investigated in a single study. Nagao et al. studied seven serum antioxidant micronutrients in 62 patients with OLP and 248 healthy control subjects, finding higher levels only of retinol (vitamin A) among the OLP patients; no significant differences were found for the other micronutrients, such as $\alpha$-tocopherol, zeaxanthin and lutein, cryptoxanthin, lycopene, $\alpha$-carotene, and $\beta$-Carotene [49]. Rezazadeh et al. studied five trace elements in 40 patients with OLP and 40 healthy control subjects, finding higher levels only of magnesium $(\mathrm{Mg})$ among the OLP patients; no significant differences were found for the other trace elements, including calcium $(\mathrm{Ca})$, iron $(\mathrm{Fe})$, zinc $(\mathrm{Zn})$, and copper $(\mathrm{Cu})$ [48].

\subsection{Total Redox Status}

3.6.1. Total Antioxidant Capacity (TAC). Seven independent publications (267 patients) were measured on salivary TAC levels and found increased levels of TAC in six [16, 29, 31, $32,34,50]$, while there was no significant difference in one study [30]. Overall, TAC in saliva showed a significant decrease in OLP (effect size -2.03, 95\% CI -3.03, -1.03), with statistically significant heterogeneity $\left(P<0.00001, I^{2}=95 \%\right)$. TAC in the plasma/serum from OLP patients was assessed in four studies (121 patients) and showed a significant decrease in all four (effect size -2.87, 95\% CI -4.56, -1.19), with statistically significant heterogeneity $\left(P<0.00001, I^{2}\right.$ $=95 \%)[15,29,30,50]$.

3.7. Subgroup Analysis. Subgroup analyses in salivary TAC and MDA studies were performed as categorized by race (Asian vs. European). The effect sizes are shown in Tables 3 and 4. From the results, salivary MDA studies showed a significantly larger effect size in Asians than in Europeans $(P=0.008)$. Subgroup analyses in salivary TAC studies showed no significant difference between Asians and Europeans $(P=0.42)$.

3.8. Sensitivity Analysis. The method of excluding the studies one by one was adopted to find if there was any research affecting the stability. The results of salivary TAC and MDA are shown in Tables 5 and 6. As shown in the tables, after excluding each single study, the salivary TAC and MDA were not significantly influenced and the $I^{2}$ also did not change remarkably.

\section{Discussion}

Our study was aimed at clarifying and quantifying the oxidative stress and antioxidant markers in the saliva and serum/plasma of OLP. The reviewed data and the results of our meta-analyses point to a role for significantly increased oxidative stress indicators and decreased antioxidant markers in individuals with OLP compared to healthy controls.

Oxidative stress is caused by an imbalance between prooxidant substances (such as reactive oxygen species (ROS) and reactive nitrogen species (RNS)) and the ability of the antioxidant system (enzymatic or nonenzymatic 


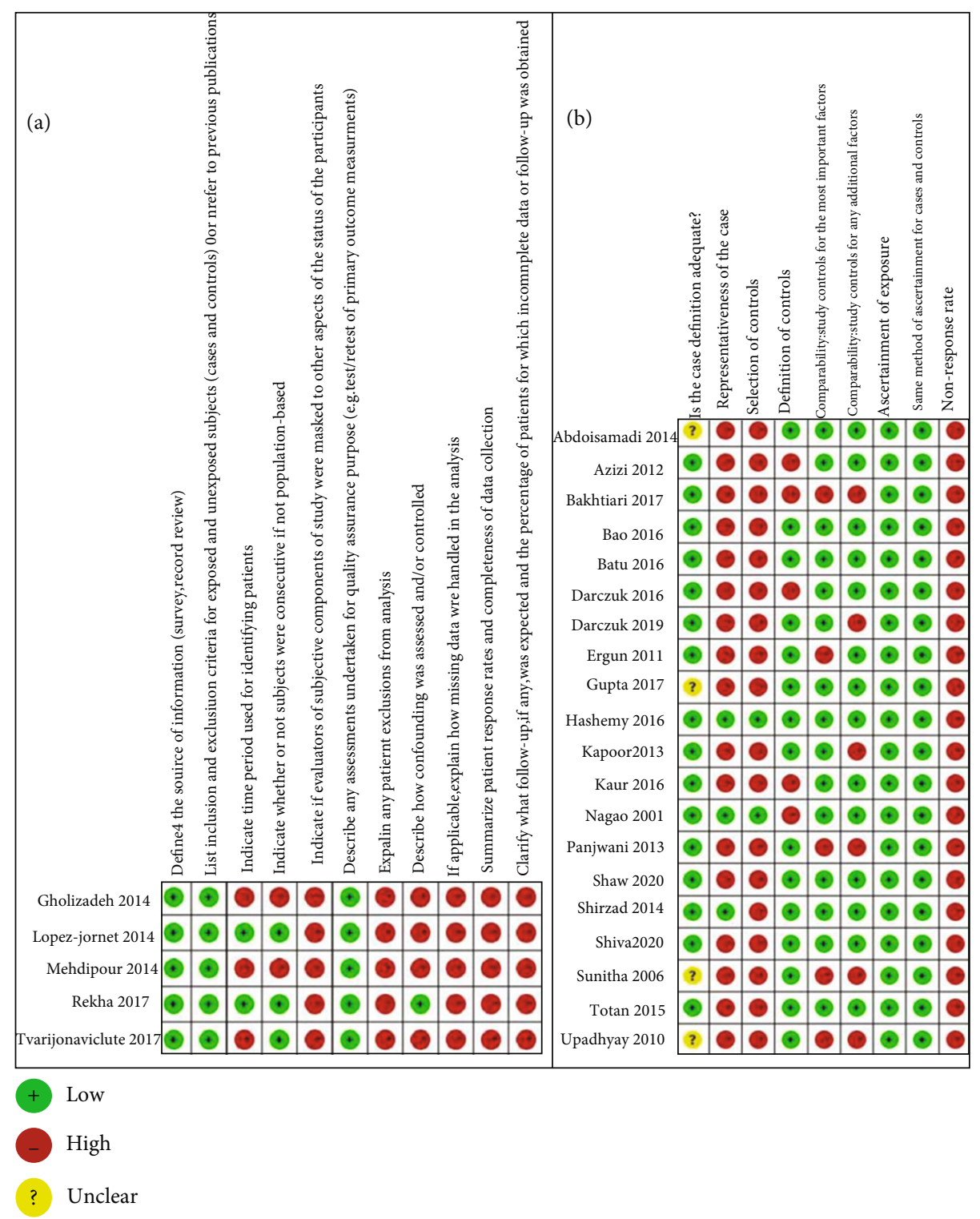

Figure 6: Bias risk of the included studies.

antioxidants) [51-54] (Figure 7). ROS are generated from multiple sources, both endogenous and exogenous. The oral cavity is a critical site for oxidative stress, and exogenous sources include oral tissue exposure to thermal, chemical, and microbial stimuli. Many behavioral factors are also conducive to the accumulation of exogenous ROS (smoking, alcohol use, and chewing betel nuts) and are also related to malignant transformation of OLP [55]. Endogenous sources refer to chronic or acute infections in oral, such as periodontitis and OLP [56]. Inflammatory cells are known to produce large amounts of ROS, and ROS in turn amplifies the inflammatory response [50].

ROS causes oxidative damage to the tissues via multiple mechanisms, including DNA damage, lipid peroxidation (LPO) damage, and protein oxidation [57-59]. 8-OHdG, both the most abundant and most investigated biomarker of oxidant-induced DNA damage, has mutagenic properties and is a risk factor for the development of cancer [60].
Although only two studies reported 8-OHdG, both of them found higher $8-\mathrm{OHdG}$ in the saliva and serum from OLP patients [29, 33]. ROS has a short half-life, making direct detection difficult. Measurement of secondary products such as LPO is suggested as a feasible option to evaluate oxidative damage. The most studied marker in the saliva of OLP patients was MDA, which is an end product of LPO and can reflect the degree of cellular damage [50, 56, 61]. From our meta-analysis, we found the level of MDA markedly increased in both the saliva and serum/plasma of OLP patients. However, controversy arises in the use of MDA levels as optimal representation for oxidative stress as it could be a nonspecific product of LPO [62]. AOPP is a cross-linking protein product modified by the interaction of free radicals and a novel marker of oxidant-mediated protein damage [63]. Our meta-analysis showed higher salivary AOOP in OLP cases than in healthy controls [28, 36]. Consistent with other reviews, we also found that the level of $\mathrm{NO}$ 
TABLE 3: Subgroup analysis of TAC (saliva).

\begin{tabular}{lcccccc}
\hline Subgroup & & $N$ & SMD $(95 \%$ CI $)$ & $P$-heterogeneity & $I^{2}(\%)$ & $P$-interaction \\
\hline \multirow{2}{*}{ Race } & Asian & 4 & $-2.41(-3.95,-0.86)$ & $<0.00001$ & 98 & 0.42 \\
& European & 3 & $-1.55(-2.93,-0.17)$ & $<0.00001$ & 98 & \\
\hline
\end{tabular}

TABLE 4: Subgroup analysis of MDA (saliva).

\begin{tabular}{lcccccc}
\hline Subgroup & & $N$ & SMD $(95 \%$ CI $)$ & $P$-heterogeneity & $I^{2}(\%)$ & $P$-interaction \\
\hline \multirow{2}{*}{ Race } & Asian & 5 & $1.30(0.76,1.85)$ & $<0.00001$ & 83 & 0.008 \\
& European & 4 & $0.92(0.62,1.21)$ & $<0.00001$ & 100 & 008 \\
\hline
\end{tabular}

TABLE 5: Sensitivity analysis of TAC (saliva) using the method of eliminating literature one by one.

\begin{tabular}{lccc}
\hline Deleted article & $I^{2}(\%)$ & $P$ & SMD $(95 \%$ CI $)$ \\
\hline Abdolsamadi & 93 & $<0.00001$ & $-1.67(-2.59,-0.74)$ \\
Azizi & 95 & $<0.00001$ & $-2.01(-3.20,-0.81)$ \\
Darczuk & 96 & $<0.00001$ & $-2.10(-3.34,-0.87)$ \\
Ergun & 95 & $<0.00001$ & $-2.33(-3.39,-1.27)$ \\
Lopez-Jornet & 93 & $<0.00001$ & $-2.33(-3.31,-1.35)$ \\
Shirzad & 95 & $<0.00001$ & $-1.89(-2.98,-0.80)$ \\
Totan & 95 & $<0.00001$ & $-1.91(-3.01,-0.80)$ \\
\hline
\end{tabular}

in OLP patients was higher than that in healthy controls [19, 64]. In recent years, a growing number of studies have confirmed that NO could be involved in OLP pathogenesis [65, 66]. The reason is that it is a free radical gas acting as a "double-edged sword." Low levels of NO are associated with hemostatic actions, while high amounts of NO can affect the ability of cells to kill bacteria, viruses, and protozoans as well as tumor cells and then participate in inflammatory and immunological disorders $[40,66,67]$.

Moreover, in our meta-analysis, lower levels of antioxidants were found in OLP patients, possibly reflecting a weakness of the defense against oxidative damage in them. TAC as an integrated parameter may serve as a better marker instead of examining individual antioxidants [68-70]. In our report, the total antioxidative activity/status/capacity (TAA/TAS/TAC) was found to be decreased in patients with OLP, which may suggest that the antioxidant defense system was inhibited. UA is an important antioxidant in saliva and plasma with the free radical scavenging capacity $[71,72]$. Our research showed that the level of UA markedly decreased in both the saliva and serum/plasma of OLP patients. Vitamin is a crucial factor in proper innate and acquired immune responses, which triggers the expression of the genes encoding the proteins involved in immune response [18]. Vit $\mathrm{A}$ and $\mathrm{E}$ inhibit the lipid peroxidation of the cell membrane, whereas Vit $\mathrm{C}$ functions as a cofactor for many enzymes. Besides, Vit $\mathrm{C}$ also helps reproduce Vit E [73]. Some researchers considered vitamins $C$ and $E$ suitable biomarkers to predict OLP [74]. Our study on Vit C in saliva showed a significantly lower level in OLP patients
TABLE 6: Sensitivity analysis of MDA (saliva) using the method of eliminating literature one by one.

\begin{tabular}{lccc}
\hline Deleted article & $I^{2}(\%)$ & $P$ & SMD $(95 \%$ CI $)$ \\
\hline Abdolsamadi & 95 & $<0.00001$ & $2.54(1.50,3.58)$ \\
Batu & 94 & $<0.00001$ & $2.61(1.64,3.58)$ \\
Darczuk & 95 & $<0.00001$ & $2.35(1.33,3.37)$ \\
Ergun & 95 & $<0.00001$ & $2.58(1.59,3.57)$ \\
Kaur & 90 & $<0.00001$ & $1.87(1.18,2.55)$ \\
Lopez-Jornet & 94 & $<0.00001$ & $2.16(1.26,3.06)$ \\
Rekha & 95 & $<0.00001$ & $2.39(1.37,3.42)$ \\
Shirzad & 95 & $<0.00001$ & $2.51(1.47,3.55)$ \\
Totan & 95 & $<0.00001$ & $2.47(1.43,3.52)$ \\
\hline
\end{tabular}

than in controls, while there was no significant difference in Vit A and E. However, considering the poor specificity of mechanisms and the strong dependency on diet limits, we think that vitamins could not be regarded as biomarkers of OLP. Moreover, some studies found that certain trace elements $(\mathrm{Mg}, \mathrm{Zn}$, etc.) may play an important role in regenerative processes against oxidative stress products in the tissues [48]. But other studies reached the opposite conclusion [49], and further research is needed in the future.

Overall, different studies in our systematic review suggested an increased oxidative stress and a decrease in antioxidant levels in OLP, thus proving that oxidative stress plays an important role in its pathophysiology. Although the etiology of OLP is unknown, it is characterized by subepithelial infiltration of $\mathrm{T}$ lymphocytes $\left(\mathrm{CD}^{+}\right.$and especially $\mathrm{CD} 8^{+}$ cells) [75]. It is believed that the generation of ROS may play a role in $\mathrm{T}$ cell immune response and then modify and cause dysregulation of immune functions by changing the balance of Th1/Th2 cytokines and increasing Th2 response $[3,76]$. Additionally, excessive reactive oxygen species (ROS) may be also correlated with a malignant transformation of OLP. The resulting elevation of oxidative stress can lead to DNA damage, protein oxidation, and lipid peroxidation. These results coupled with lack of cellular repair processes have been shown to be associated with mutation-induced carcinogenesis [53, 63, 77].

We pooled only measurements carried out on the same biological sample (saliva or plasma/serum), but the heterogeneity of most studies was high other than the studies on 


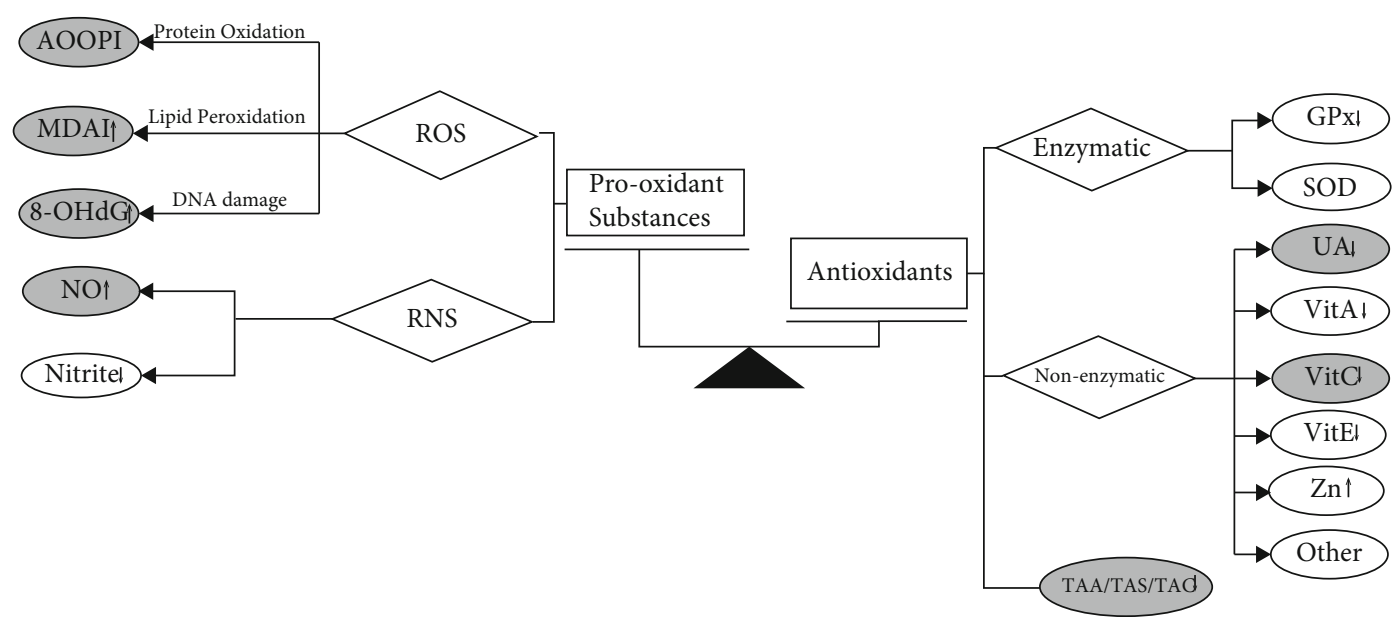

Figure 7: Schematic representation of the oxidative stress and antioxidant markers in oral lichen planus. Black arrows: markers with statistical differences in meta-analysis. Grey arrows: trend but no statistical differences. Unmarked: reported by a single study. Abbreviations: $\mathrm{ROS}=$ reactive oxygen species; RNS = reactive nitrogen species; $\mathrm{MDA}=$ malondialdehyde; $\mathrm{AOOP}=$ advanced oxidation protein product; 8-OHdG $=8$-hydroxy-deoxyguanosine; $\mathrm{NO}=$ nitric oxide; $\mathrm{GPx}=$ glutathione peroxidase; $\mathrm{SOD}=$ superoxide dismutase; $\mathrm{UA}=$ uric acid; Vit = vitamin; $\mathrm{Zn}=$ zinc; $\mathrm{TAC}=$ total antioxidative capacity.

NO in serum/plasma $\left(P=0.31, I^{2}=3 \%\right)$ and AOOP in saliva $\left(P=0.48, I^{2}=0 \%\right)$. As to the studies whose components may contribute to heterogeneity, we conducted subgroup and sensitivity analyses for salivary TAC and MDA. By subgroup analysis, we found that salivary MDA in Asians was significantly higher than that in Europeans $(P=0.008)$, while no racial difference was noted in salivary TAC $(P=0.42)$. The sensitive analysis showed that after excluding each single study, the salivary TAC and MDA and $I^{2}$ were not markedly influenced. Thus, the robustness of the results was proven. We investigated further possible causes of heterogeneity among studies (e.g., host factor, sample collection, assay methods, and others). First, in terms of host factor, the individual patient characteristics varied greatly between studies (age, gender, race, body mass index (BMI), and smoking), and the factor of antioxidant supplementation and diet might both influence biomarker levels. The type, severity, and location of OLP could cause heterogeneity. Some studies focused on patients diagnosed with erosive OLP, while others did not divide OLP into different forms. The lower concentration of antioxidant defense markers and higher levels of oxidative stress markers were found in the erosive form as compared to other forms of OLP [32, 42]. The more serious condition of oxidative stress in patients with erosive OLP seemed to reflect more intense inflammation and potential capacity for malignant transformation. The levels of these markers were associated with OLP lesion severity [28]. However, all included studies were observational and at a significant risk of bias and confounding. The limited number of the studies included made it unclear whether publication bias could be a factor in these markers. Such biases can be resolved by increasing the number of included articles in future research. The imbalance between oxidant and antioxidant systems also plays a role in other oral diseases, including recurrent aphthous stomatitis (RAS) and periodontitis [78, 79]. Not all authors gave clear details on these points. Finally, different assay methods, testing equipment, sample collection status, and reagent kits in the included studies could cause measurement bias.

The strengths of this meta-analysis lie in our extensive and comprehensive search to identify all studies on the association of oxidative stress and antioxidant markers with OLP, rigorous evaluation and one-by-one analysis, and further statistical processing of the data through quantitative synthesis to reach comprehensive conclusions. Meanwhile, a relatively objective evaluation of oxidative stress and antioxidant markers in OLP could be performed by analyzing the heterogeneity of different studies.

Our meta-analysis showed that oxidative stress and antioxidant markers may be the potential biomarkers to diagnose OLP. Increase in oxidants and decrease in antioxidants might be an indicator of OLP occurrence. As the oral cavity is the start of the digestion system, saliva acts as the first line of defense against OS with abundant antioxidants, such as UA, albumin, and ascorbic acid [80]. We found that the markers in saliva and blood have the same variation tendency. Similar to our study, Ergun et al. showed a significant correlation between oxidant and antioxidant salivary and serum levels [30]. These results suggest that saliva can be considered a reliable medium in assessing oxidative stress levels, which makes it possible to diagnose OLP noninvasively. As a better substitute for blood and urine samples, saliva can serve as a diagnostic fluid and presents many advantages: it is noninvasive, safe, painless, and easy to be collected [35]. In addition, salivary OS markers represent the state of local oral oxidative stress, which can better reflect the real situation of local oral microenvironment. However, using saliva to measure oxidative stress in OLP also has some limitations. There is still a lack of unified and standardized processes for saliva collection time, and whether it needs to be stimulated or centrifuged after sampling, its storage temperature and time, and analysis methods all remain elusive [31]. 
This meta-analysis has several limitations. Firstly, the number of included studies on each marker with high heterogeneity is limited $(n<10)$, and more homogeneous studies on oxidative stress in OLP are needed. Secondly, the quality of the above literature was generally not high and had small sizes of the study groups. To further evaluate the role of oxidative and antioxidative stress markers in OLP, large and strict quality control studies in various regions are necessary. Thirdly, thus far, most published studies have assessed oxidant-antioxidant status in patients with OLP in comparison with healthy individuals and the correlation between the markers and the degree of OLP lesions. However, the diagnostic utility of salivary redox biomarkers has not been truly validated in OLP diagnosis. Estimation of specificity, sensitivity, predictive values, ROC analysis or cluster analysis, etc. has not been reported when attempting to use biomarkers as a diagnostic/prognostic markers in OLP. Further studies in this direction will help to find a reliable and unambiguous diagnostic or prognostic marker among the OS and antioxidant markers, which could be used as a therapeutic target in clinical practice. Last but not least, although the association between OLP and OS is well established and the patients with increased free radicals in serum and saliva and decreased activity of antioxidants support this thesis, it is not clear whether OS is the cause or a result of OLP. Further studies are required to investigate molecular mechanisms underlying the etiology of these pathologies and understand in depth the potential role of OS in OLP.

\section{Conclusions}

In conclusion, studies on saliva and plasma/serum biomarkers related to oxidative stress and antioxidants in OLP patients provide evidence suggestive of a significant increase in NO and MDA in saliva and plasma/serum and 8-OHdG and AOOP in saliva, as well as a significant decrease in TAC and UA in saliva and plasma/serum and Vit $C$ in saliva. To sum up, NO, 8-OHdG, AOOP, TAC, and UA may be the potential biomarkers for measuring the effect imbalance of antioxidant-oxidative stress in OLP patients. Nevertheless, there are only a limited number of studies on some markers and with high heterogeneity; more homogeneous studies on oxidative stress in OLP are needed. Moreover, further studies are also needed, including how to explain the link by examining the underlying mechanisms and whether antioxidant therapy could be developed as a new significant therapy for decreasing inflammation and producing positive, long-term effects on OLP.

\section{Data Availability}

The data used to support the findings of this study are included within the article.

\section{Conflicts of Interest}

The authors declare that there is no conflict of interest regarding the publication of this article.

\section{Authors' Contributions}

Yuan Fan conceptualized and designed the study. Jia Wang and Jingjing Yang performed literature search, screened appropriate articles, and conducted data processing. Chen Wang and Zhibai Zhao drafted the manuscript and were involved in the critical revision of the manuscript. All authors read and approved the final version of the manuscript.

\section{Acknowledgments}

This work was supported by the National Nature Science Foundation of China (Grant No. 81970941), the Priority Academic Program Development of Jiangsu Higher Education Institutions (PAPD, 2018-87), and the Special Fund of Jiangsu Provincial Key Research and Development Project (Social Development) (Grant No. BE2021723).

\section{References}

[1] B. E. McCartan and C. M. Healy, "The reported prevalence of oral lichen planus: a review and critique," Journal of Oral Pathology \& Medicine, vol. 37, no. 8, pp. 447-453, 2008.

[2] M. R. Payeras, K. Cherubini, M. A. Figueiredo, and F. G. Salum, "Oral lichen planus: focus on etiopathogenesis," Archives of oral biology, vol. 58, no. 9, pp. 1057-1069, 2013.

[3] M. A. Gonzalez-Moles, C. Scully, and J. A. Gil-Montoya, "Oral lichen planus: controversies surrounding malignant transformation," Oral diseases, vol. 14, no. 3, pp. 229-243, 2008.

[4] C. Scully, M. Beyli, M. C. Ferreiro et al., "Update on oral lichen planus: etiopathogenesis and management," Critical reviews in oral biology and medicine, vol. 9, no. 1, pp. 86-122, 1998.

[5] V. Bhatia and S. Sharma, "Role of mitochondrial dysfunction, oxidative stress and autophagy in progression of Alzheimer's disease," Journal of the neurological sciences, vol. 421, 2021.

[6] F. Guarneri, P. Custurone, V. Papaianni, and S. Gangemi, "Involvement of RAGE and oxidative stress in inflammatory and infectious skin diseases," Antioxidants, vol. 10, no. 1, p. 82, 2021.

[7] C. Izzo, P. Vitillo, P. di Pietro et al., "The role of oxidative stress in cardiovascular aging and cardiovascular diseases," Life, vol. 11, no. 1, p. 60, 2021

[8] P. Wójcik, A. Gęgotek, N. Žarković, and E. Skrzydlewska, "Oxidative stress and lipid mediators modulate immune cell functions in autoimmune diseases," International Journal of Molecular Sciences, vol. 22, no. 2, p. 723, 2021.

[9] D. Miricescu, A. Totan, B. Calenic et al., "Salivary biomarkers: relationship between oxidative stress and alveolar bone loss in chronic periodontitis," Acta odontologica Scandinavica, vol. 72, no. 1, pp. 42-47, 2014.

[10] I. Peluso and A. Raguzzini, "Salivary and urinary total antioxidant capacity as biomarkers of oxidative stress in humans," Pathology research international, vol. 2016, Article ID 5480267, 14 pages, 2016.

[11] W. Dröge, "Free radicals in the physiological control of cell function," Physiological Reviews, vol. 82, no. 1, pp. 47-95, 2002.

[12] B. S. Abrahams and D. H. Geschwind, "Advances in autism genetics: on the threshold of a new neurobiology," Nature reviews. Genetics, vol. 9, no. 5, pp. 341-355, 2008. 
[13] B. S. Abrahams and D. H. Geschwind, "Connecting genes to brain in the autism spectrum disorders," Archives of Neurology, vol. 67, no. 4, pp. 395-399, 2010.

[14] W. R. McGinnis, "Oxidative stress in autism," Alternative therapies in health and medicine, vol. 10, 2004.

[15] R. B. Upadhyay, S. Carnelio, R. P. Shenoy, P. Gyawali, and M. Mukherjee, "Oxidative stress and antioxidant defense in oral lichen planus and oral lichenoid reaction," Scandinavian journal of clinical and laboratory investigation, vol. 70, no. 4, pp. 225-228, 2010.

[16] A. Shirzad, M. Pouramir, M. Seyedmajidi, N. Jenabian, A. Bijani, and M. Motallebnejad, "Salivary total antioxidant capacity and lipid peroxidation in patients with erosive oral lichen planus," Journal of dental research, dental clinics, dental prospects, vol. 8, no. 1, pp. 35-39, 2014.

[17] S. L. Sankari, N. A. Babu, E. Rajesh, and M. Kasthuri, "Apoptosis in immune-mediated diseases," Journal of pharmacy \& bioallied sciences, vol. 7, Supplement 1, pp. S200-S202, 2015.

[18] N. Gholizadeh and N. Sheykhbahaei, "Micronutrients profile in oral lichen planus: a review literature," Biological trace element research, vol. 199, no. 3, pp. 912-924, 2021.

[19] J. S. M. Humberto, J. V. Pavanin, M. J. A. Rocha, and A. C. F. Motta, "Cytokines, cortisol, and nitric oxide as salivary biomarkers in oral lichen planus: a systematic review," Brazilian oral research, vol. 32, 2018.

[20] S. S. Mishra and T. N. Uma Maheswari, "Evaluation of oxidative stress in oral lichen planus using malonaldehyde: a systematic review," Journal of Dermatology and Dermatologic Surgery, vol. 18, no. 1-2, pp. 2-7, 2014.

[21] J. P. Higgins, J. Thomas, J. Chandler et al., Cochrane Handbook for Systematic Reviews of Intervensions version 6.2 (updated February 2021), Cochrane, 2021.

[22] A. Liberati, D. G. Altman, J. Tetzlaff et al., "The PRISMA statement for reporting systematic reviews and meta-analyses of studies that evaluate healthcare interventions: explanation and elaboration," BMJ, vol. 339, no. jul21 1, p. b2700, 2009.

[23] A. Stang, "Critical evaluation of the Newcastle-Ottawa scale for the assessment of the quality of nonrandomized studies in meta-analyses," European journal of epidemiology, vol. 25, no. 9, pp. 603-605, 2010.

[24] A. Rostom, C. Dubé, A. Cranney et al., "Celiac Disease. Rockville (MD): Agency for Healthcare Research and Quality (US); 2004 Sep. (Evidence Reports/Technology Assessments, No. 104.) Appendix D. Quality Assessment Forms," http://www .ncbi.nlm.nih.gov/books/NBK35156.

[25] S. P. Hozo, B. Djulbegovic, and I. Hozo, "Estimating the mean and variance from the median, range, and the size of a sample," BMC Medical Research Methodology, vol. 5, no. 1, 2005.

[26] J. P. Higgins, S. G. Thompson, J. J. Deeks, and D. G. Altman, "Measuring inconsistency in meta-analyses," BMJ, vol. 327, no. 7414, pp. 557-560, 2003.

[27] J. P. Ioannidis and T. A. Trikalinos, "The appropriateness of asymmetry tests for publication bias in meta-analyses: a large survey," CMAJ, vol. 176, no. 8, pp. 1091-1096, 2007.

[28] Ş. Batu, D. Ofluoğlu, S. Ergun et al., "Evaluation of prolidase activity and oxidative stress in patients with oral lichen planus and oral lichenoid contact reactions," Journal of oral pathology \& medicine, vol. 45, no. 4, pp. 281-288, 2016.

[29] A. Totan, D. Miricescu, I. Parlatescu, M. Mohora, and M. Greabu, "Possible salivary and serum biomarkers for oral lichen planus," Biotechnic \& histochemistry, vol. 90, no. 7, pp. 552-558, 2015.

[30] S. Ergun, S. C. Troşala, S. Warnakulasuriya et al., "Evaluation of oxidative stress and antioxidant profile in patients with oral lichen planus," Journal of Oral Pathology and Medicine, vol. 40, no. 4, pp. 286-293, 2011.

[31] H. Abdolsamadi, N. Rafieian, M. T. Goodarzi et al., "Levels of salivary antioxidant vitamins and lipid peroxidation in patients with oral lichen planus and healthy individuals," Chonnam medical journal, vol. 50, no. 2, pp. 58-62, 2014.

[32] D. Darczuk, W. Krzysciak, P. Vyhouskaya et al., "Salivary oxidative status in patients with oral lichen planus," Journal of physiology and pharmacology, vol. 67, no. 6, pp. 885-894, 2016.

[33] J. Kaur, C. Politis, and R. Jacobs, "Salivary 8-hydroxy-2-deoxyguanosine, malondialdehyde, vitamin $\mathrm{C}$, and vitamin $\mathrm{E}$ in oral pre-cancer and cancer: diagnostic value and free radical mechanism of action," Clinical oral investigations, vol. 20, no. 2, pp. 315-319, 2016.

[34] P. Lopez-Jornet, A. Martinez-Canovas, and A. Pons-Fuster, "Salivary biomarkers of oxidative stress and quality of life in patients with oral lichen planus," Geriatrics \& gerontology international, vol. 14, no. 3, pp. 654-659, 2014.

[35] V. R. Rekha, S. Sunil, and R. Rathy, "Evaluation of oxidative stress markers in oral lichen planus," Journal of oral and maxillofacial pathology, vol. 21, no. 3, pp. 387-393, 2017.

[36] A. Tvarijonaviciute, C. Aznar-Cayuela, C. P. Rubio, J. J. Ceron, and P. López-Jornet, "Evaluation of salivary oxidate stress biomarkers, nitric oxide and C-reactive protein in patients with oral lichen planus and burning mouth syndrome," Journal of oral pathology \& medicine, vol. 46, no. 5, pp. 387-392, 2017.

[37] C. Kapoor, V. Wadhwan, S. Vaidya, and S. Malik, “Triology of nitric oxide, mast cell, and stress in pathogenesis of oral lichen planus," Journal of oral and maxillofacial pathology, vol. 17, no. 2, pp. 156-162, 2013.

[38] S. Panjwani, A. Bagewadi, V. Keluskar, R. Malik, S. Rai, and D. Misra, "Estimation and comparison of levels of salivary nitric oxide in patients with oral lichen planus and controls," International journal of preventive medicine, vol. 4, no. 6 , pp. 710-714, 2013.

[39] A. Shiva, S. Arab, S. J. Mousavi, A. Zamanian, and A. Maboudi, "Serum and salivary level of nitric oxide (NOx) and CRP in oral lichen planus (OLP) patients," Journal of dentistry, vol. 21, no. 1, pp. 6-11, 2020.

[40] M. Mehdipour, A. Taghavi Zenouz, A. Bahramian, N. Gholizadeh, and M. Boorghani, "Evaluation of serum nitric oxide level in patients with oral lichen planus," Journal of dentistry, vol. 15, no. 2, pp. 48-51, 2014.

[41] M. Sunitha and S. Shanmugam, "Evaluation of salivary nitric oxide levels in oral mucosal diseases: a controlled clinical trial," Indian journal of dental research, vol. 17, no. 3, pp. 117-120, 2006.

[42] D. Darczuk, W. Krzyściak, B. Bystrowska et al., "The relationship between the concentration of salivary tyrosine and antioxidants in patients with oral lichen planus," Oxidative medicine and cellular longevity, vol. 2019, Article ID 5801570, 11 pages, 2019.

[43] A. Gupta, R. P. Mohan, S. Gupta, S. S. Malik, S. Goel, and N. Kamarthi, "Roles of serum uric acid, prolactin levels, and psychosocial factors in oral lichen planus," Journal of oral science, vol. 59, no. 1, pp. 139-146, 2017.

[44] H. Shaw, A. Konidena, A. Malhotra, N. Yumnam, F. Farooq, and V. Bansal, "Psychological status and uric acid levels in oral 
lichen planus patients - a case- control study," Indian journal of dental research, vol. 31, no. 3, pp. 368-375, 2020.

[45] S. Bakhtiari, P. Toosi, S. Samadi, and M. Bakhshi, "Assessment of uric acid level in the saliva of patients with oral lichen planus," Medical principles and practice, vol. 26, no. 1, pp. 57-60, 2017.

[46] Z. X. Bao, X. W. Yang, J. Shi, and L. X. Liu, "Serum zinc levels in 368 patients with oral mucosal diseases: a preliminary study," Medicina oral, patologia oral y cirugia bucal, vol. 21, no. 3, pp. e335-e340, 2016.

[47] N. Gholizadeh, M. Mehdipour, Najafi Sh, A. Bahramian, Garjani Sh, and H. Khoeini Poorfar, "Evaluation of the serum zinc level in erosive and non-erosive oral lichen planus," Journal of dentistry, vol. 15, no. 2, pp. 52-56, 2014.

[48] F. Rezazadeh, S. Salehi, and M. Rezaee, "Salivary level of trace element in oral lichen planus, a premalignant condition," Asian Pacific journal of cancer prevention, vol. 20, no. 7, pp. 2009-2013, 2019.

[49] T. Nagao, S. Warnakulasuriya, N. Ikeda et al., "Serum antioxidant micronutrient levels in oral lichen planus," Journal of oral pathology \& medicine, vol. 30, no. 5, pp. 264-267, 2001.

[50] A. Azizi and F. Farshchi, "Comparison of salivary and plasma antioxidant levels in lichen planus patients and healthy subjects," Journal of oral pathology \& medicine, vol. 41, no. 7, pp. 524-526, 2012.

[51] M. Battino, M. S. Ferreiro, I. Gallardo, H. N. Newman, and P. Bullon, "The antioxidant capacity of saliva," Journal of clinical periodontology, vol. 29, no. 3, pp. 189-194, 2002.

[52] S. Briganti and M. Picardo, "Antioxidant activity, lipid peroxidation and skin diseases. What's new," Journal of the European Academy of Dermatology and Venereology, vol. 17, no. 6, pp. 663-669, 2003.

[53] P. Chaiyarit, N. Ma, Y. Hiraku et al., "Nitrative and oxidative DNA damage in oral lichen planus in relation to human oral carcinogenesis," Cancer science, vol. 96, no. 9, pp. 553-559, 2005.

[54] I. Hovatta, J. Juhila, and J. Donner, "Oxidative stress in anxiety and comorbid disorders," Neuroscience research, vol. 68 , no. 4 , pp. 261-275, 2010.

[55] T. Nosratzehi, "Oral lichen planus: an overview of potential risk factors, biomarkers and treatments," Asian Pacific journal of cancer prevention, vol. 19, no. 5, pp. 1161-1167, 2018.

[56] D. G. Aly and R. S. Shahin, "Oxidative stress in lichen planus," Acta dermatovenerologica Alpina, Pannonica, et Adriatica, vol. 19, no. 1, pp. 3-11, 2010.

[57] F. A. Akalın, E. Baltacıoğlu, A. Alver, and E. Karabulut, "Lipid peroxidation levels and total oxidant status in serum, saliva and gingival crevicular fluid in patients with chronic periodontitis," Journal of clinical periodontology, vol. 34, no. 7, pp. 558565, 2007.

[58] I. L. Chapple and J. B. Matthews, "The role of reactive oxygen and antioxidant species in periodontal tissue destruction," Periodontology 2000, vol. 43, no. 1, pp. 160-232, 2007.

[59] I. L. Chapple, M. R. Milward, and T. Dietrich, "The prevalence of inflammatory periodontitis is negatively associated with serum antioxidant concentrations," The Journal of nutrition, vol. 137, no. 3, pp. 657-664, 2007.

[60] T. Paz-Elizur, Z. Sevilya, Y. Leitner-Dagan, D. Elinger, L. C. Roisman, and Z. Livneh, "DNA repair of oxidative DNA damage in human carcinogenesis: potential application for cancer risk assessment and prevention," Cancer Letters, vol. 266, no. 1 , pp. $60-72,2008$.
[61] B. Vlková, P. Stanko, G. Minárik et al., "Salivary markers of oxidative stress in patients with oral premalignant lesions," Archives of oral biology, vol. 57, no. 12, pp. 1651-1656, 2012.

[62] B. Halliwell and M. Whiteman, "Measuring reactive species and oxidative damage in vivo and in cell culture: how should you do it and what do the results mean?," British journal of pharmacology, vol. 142, no. 2, pp. 231-255, 2004.

[63] R. Hanafi, I. Anestopoulos, G. P. Voulgaridou et al., "Oxidative stress based-biomarkers in oral carcinogenesis: how far have we gone?" Current Molecular Medicine, vol. 12, no. 6, pp. 698-703, 2012.

[64] D. Uğar-Çankal and N. Ozmeric, "A multifaceted molecule, nitric oxide in oral and periodontal diseases," Clinica Chimica Acta, vol. 366, no. 1-2, pp. 90-100, 2006.

[65] C. J. Lowenstein and S. H. Snyder, "Nitric oxide, a novel biologic messenger," Cell, vol. 70, no. 5, pp. 705-707, 1992.

[66] M. Ohashi, M. Iwase, and M. Nagumo, "Elevated production of salivary nitric oxide in oral mucosal diseases," Journal of oral pathology \& medicine, vol. 28, no. 8, pp. 355-359, 1999.

[67] P. A. Brennan, T. Umar, M. Palacios-Callender et al., "A study to assess inducible nitric oxide synthase expression in oral lichen planus," Journal of oral pathology \& medicine, vol. 29, no. 6, pp. 249-254, 2000.

[68] A. Ghiselli, M. Serafini, F. Natella, and C. Scaccini, "Total antioxidant capacity as a tool to assess redox status: critical view and experimental data," Free radical biology \& medicine, vol. 29, no. 11, pp. 1106-1114, 2000.

[69] M. Serafini and D. Del Rio, "Understanding the association between dietary antioxidants, redox status and disease: is the total antioxidant capacity the right tool?," Redox report, vol. 9, no. 3, pp. 145-152, 2004.

[70] M. Serafini, D. Villano, G. Spera, and N. Pellegrini, "Redox molecules and cancer prevention: the importance of understanding the role of the antioxidant network," Nutrition and cancer, vol. 56, no. 2, pp. 232-240, 2006.

[71] C. Gersch, S. P. Palii, K. M. Kim, A. Angerhofer, R. J. Johnson, and G. N. Henderson, "Inactivation of nitric oxide by uric acid," Nucleosides, nucleotides \& nucleic acids, vol. 27, no. 8, pp. 967-978, 2008.

[72] J. Kun, A. Perkecz, L. Knie et al., “TRPA1 receptor is upregulated in human oral lichen planus," Oral diseases, vol. 23, no. 2, pp. 189-198, 2017.

[73] K. L. Keller and N. A. Fenske, "Uses of vitamins A, C, and E and related compounds in dermatology: a review," Journal of the American Academy of Dermatology, vol. 39, no. 4, pp. 611-625, 1998.

[74] B. Rai, J. Kaur, R. Jacobs, and J. Singh, "Possible action mechanism for curcumin in pre-cancerous lesions based on serum and salivary markers of oxidative stress," Journal of oral science, vol. 52, no. 2, pp. 251-256, 2010.

[75] A. Khan, C. S. Farah, N. W. Savage, L. J. Walsh, D. J. Harbrow, and P. B. Sugerman, "Th1 cytokines in oral lichen planus," Journal of oral pathology \& medicine, vol. 32, no. 2, pp. 7783, 2003.

[76] E. H. van der Meij, H. Mast, and I. van der Waal, "The possible premalignant character of oral lichen planus and oral lichenoid lesions: a prospective five-year follow-up study of 192 patients," Oral oncology, vol. 43, no. 8, pp. 742-748, 2007.

[77] N. Mortazavi, "Role of oxidative stress in malignant transformation of oral lichen planus," Oral oncology, vol. 49, no. 12, pp. e41-e42, 2013. 
[78] J. Bagan, G. Saez, C. Tormos et al., "Oxidative stress and recurrent aphthous stomatitis," Clinical oral investigations, vol. 18, no. 8, pp. 1919-1923, 2014.

[79] G. Z. Racz, K. Kadar, A. Foldes et al., "Immunomodulatory and potential therapeutic role of mesenchymal stem cells in periodontitis," Journal of physiology and pharmacology, vol. 65, no. 3, pp. 327-339, 2014.

[80] A. W. H. Alamir, G. Arakeri, S. Patil et al., "Association of nitric oxide with oral lichen planus," Journal of oral pathology \& medicine, vol. 48, no. 5, pp. 345-350, 2019. 